\title{
Load with Salmonella spp. in Fattening Pigs after Weaning Until to Fattening
}

\author{
Zoriţa Maria COCORA ${ }^{1 *}$, Ioan ŢIBRU ${ }^{1}$ \\ Banat's University of Agricultural Science and Veterinary Medicine Timisoara "King Michael of Romania", \\ Faculty of Veterinary Medicine, 300645, Calea Aradului, no 119, Timisoara, Romania \\ *Corresponding author: zoritzacocora@yahoo.com
}

Bulletin UASVM Veterinary Medicine 72(2) / 2015,

Print ISSN 1843-5270; Electronic ISSN 1843-5378

DOI:10.15835/buasvmcn-vm: 11354

\begin{abstract}
The aim of the present study was to monitor the carrier status for Salmonella spp. in piglets from two farms (farm A and farm B), collecting samples from piglets after weaning until fattening, on the same animals. For this purpose 60 faecal samples were collected immediately after weaning until tansfer to fattening facilities.

By analyzing faecal samples collected from both farms (farm A and B), a 50\% Salmonella spp. load was observed in weaned piglets, and an increase by $7 \%$ to young stock.

Identification and isolation of Salmonella was carried out by EN ISO 6579: 2003 method followed by serum agglutination, and to isolate Salmonella spp. serovars API 20E method was used.
\end{abstract}

Keywords: contamination, piglets, Salmonella spp., samples, serovars

\section{INTRODUCTION}

Weaning stress and horizontal transmission occurred in the nursery were the important factors of increasing the prevalence in the nursery. The most important stress factors are likely to be: a change in feed, the commingling of litter, a piglet's being deprived of the antibodies found in sow's milk before the activation of its own immune response (Kranker et al., 2003).

\section{AIMS AND OBJECTIVES}

The objectives of this study was to monitor the transmission possibility of Salmonella, by analyzing samples of piglets from weaning to fattening, thus making an assessment of the risk factors involved in the introduction of Salmonella at each stage of the production chain.

\section{MATERIALS AND METHODS}

The study was conducted in two pig production farms ( $A$ and $B$ ). A total number of 150 pigs were monitored, housed in 6 pens, from weaning for
90 days. The faecal sampling frequency was every two weeks, totalling 60 samples.

Piglets were weaned at 28 days of age, being transferred to the nursery pens within the same farm. Examination of samples was conducted in the laboratory of animal hygiene, using the bacteriological method SR EN ISO 6579/2003 and serovars were identified by method API 20E.

\section{RESULTS AND DISCUSSION}

After analyzing the collected samples (n $=60$ ) from weaned piglets in each farm a high degree of Salmonella spp. contamination was revealed, positive samples were recorded in 57\% of piglets in farm A and in $43 \%$ of piglets in farm B. Continuing examination of the collected samples, a slight increase of positive samples by $11 \%$ was observed in farm $\mathrm{A}$, before their transfer in fattening pens, while in farm $B$, the increase was only $3 \%$ of positive samples (Fig.1).

These results are due to non-compliances of disinfection and cross-contamination with faeces 


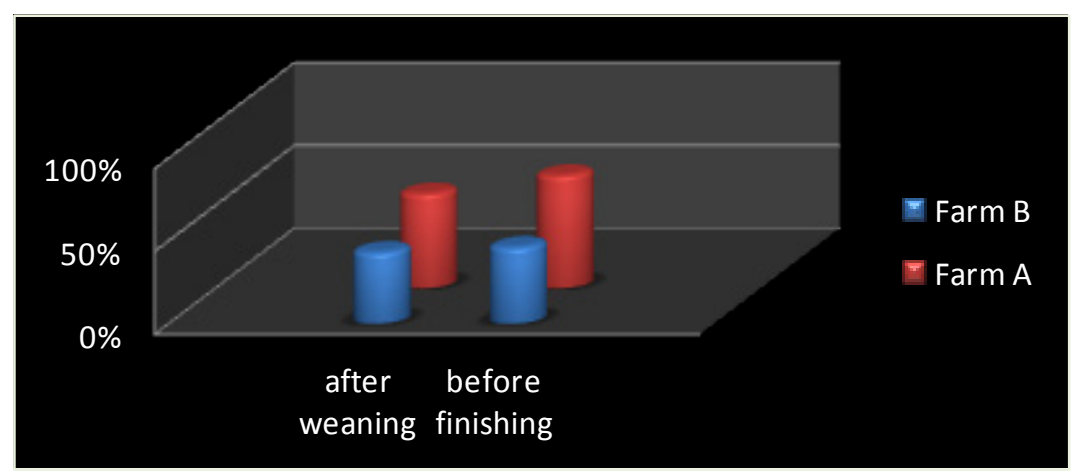

Fig. 1. The presence of Salmonella in pigs after weaning and in young pigs

from Salmonella spp. carrier piglets. Also, this increase may be due to the fact that after weaning, piglets' immunity is declining, they are deprived of the maternal milk protection, adding to this the re-grouping and ration changing, Thus, piglets become sensitive to various infections, including microorganisms of the Salmonella genus.

After analyzing the samples by API 20E method, the most common serovars isolated from weaned piglets, and from young pigs until their transfer to the fattening pens were: Salmonella Typhimurium at a rate of $59.95 \%$, followed by Salmonella Choleraesuis (31.17\%), and Salmonella spp. was found to make up $15.57 \%$ of the positive samples.

Through these analyzes, Salmonella spp. dispersion was monitored, as well as the distribution of the most common isolated Salmonella spp. serovars. Also, the role of carrier status of the animals was monitored by collecting samples from weaned piglets, and in young pigs until the age of fattening, in two different farms.

Similar results were obtained by Funk et al. (2001) and Kranker et al. (2003), where the authors found an increase in the number of pigs infected with Salmonella spp.

\section{CONCLUSION}

A load of Salmonella spp. of $57 \%$ (farm A) and $43 \%$ (farm B) was observed after weaning the piglets. Before moving piglets to fattening pens, the number of positive samples increased to $68 \%$ and $47 \%$ (farm A and B, respectively).The main serovars isolated from weaned piglets, and from young pigs until their transfer to fattening pens were: Salmonella Typhimurium (59.95\%), and Salmonella Choleraesuis (31.17\%).

Acknowledgements: This paper was published under the frame of the European Social Fund, Human Resources Development Operational Programme 2007-2013, project no. POSDRU/159/ $1.5 / S / 132765$.

\section{REFERENCES}

1. Funk JA, Davie, PR, Nichols MA (2001). Longitudinal study of Salmonella enterica in growing pigs reared in multi-site swine production systems. Vet Micro 83.

2. Kranker SL, Alban J, Boes J, Dahl J (2003). Longitudinal study of Salmonella enterica serotype Typhimurium infection in three Danish farrow-to-finish swine herds. J Clin Microbiol 41:2282-2288. 